\title{
51. Existence of Periodic Solutions of Nonlinear Hyperbolic System
}

\author{
By Yasumasa NishiURA \\ Faculty of Science, Kyoto University \\ (Communicated by Kôsaku YosidA, M. J.A., Nov. 12, 1977)
}

In this paper we discuss the problem of the existence of real periodic solutions along the characteristic lines satisfying the nonlinear hyperbolic system of equations for the unknown $U={ }^{t}\left(u_{1}, u_{2}\right)$

$$
U_{t}+A U_{x}=B U+F(U, \varepsilon) \text {, }
$$

where $A$ and $B$ are real constant matrices as follows:

$$
A=\left(\begin{array}{cc}
c_{1} & 0 \\
0 & c_{2}
\end{array}\right), \quad c_{1} \neq c_{2}, \quad B=\left(\begin{array}{ll}
a & b \\
c & d
\end{array}\right),
$$

and $F(U, \varepsilon)={ }^{t}\left(f_{1}\left(u_{1}, u_{2}, \varepsilon\right), f_{2}\left(u_{1}, u_{2}, \varepsilon\right)\right)$ is a smooth real-valued vector function and $\varepsilon$ is a real parameter. We assume that $F(U, 0)=0$ and $B$ satisfies

$$
a d \cdot \operatorname{det}(B)<0 .
$$

We write (1) in the characteristic form

$$
D U=B U+F(U, \varepsilon),
$$

where

$$
D U={ }^{t}\left(\partial u_{1} / \partial \xi, \partial u_{2} / \partial \eta\right) \text {, and } \xi=\frac{x-c_{2} t}{c_{1}-c_{2}}, \eta=\frac{x-c_{1} t}{c_{2}-c_{1}} .
$$

We note that nonlinear term $F(U, \varepsilon)$ is autonomous (i.e. not explicitly depends on $\xi, \eta)$. As for non-autonomous periodic perturbation, there is an extensive literature concerning the existence of periodic solutions of nonlinear hyperbolic equations such as [1], [2], [4], [5], [6].

In Lemmas 1 and 2 we summarize the results about the un-perturbed equation

$$
D U=B U \text {. }
$$

Under the assumption (2) of $B$, it is easy to prove those lemmas by Fourier series expansion, so we omit them. In Lemmas 3 and 4 , we reduce the problem about (3) to solve the bifurcation equation, and using an implicit function theorem we can obtain the main theorem. In the last part of this paper we show an example in population dynamics with migrational effect to which our theorem can apply. We use the following notations:

$D_{\#}$ is the space of $C^{\infty}$-periodic vector functions with $S$-periodic in $\xi$ and $T$-periodic in $\eta$ where $S$ and $T$ are constants given in Lemma 1, 
and which is topologized by the usual seminorms, $D_{\#}^{\prime}$ is the space of periodic distributions on $D_{\text {\#, }}$

$$
H_{\#}^{k}=\left\{U=^{t}\left(u_{1}, u_{2}\right) ; U \in D_{\#}^{\prime},\|U\|_{k}=\left\|u_{1}\right\|_{k}+\left\|u_{2}\right\|_{k}<+\infty\right\},
$$

where we denote the usual Sovolev $k$-norm by $\|\cdot\|_{k}$, and

$$
H_{\#}^{k}(b)=\left\{U={ }^{t}\left(u_{1}, u_{2}\right) ; U \in H_{\#}^{k},\|U\|_{k} \leqq b\right\} .
$$

Lemma 1. If $B$ satisfies (2), then linear equation (4) has periodic solutions with S-periodic in $\xi$ and T-periodic in $\eta$ where

$$
S=\frac{2 \pi}{r}, \quad T=\frac{2 \pi}{r}\left|\frac{a}{d}\right|, \quad r=\left\{\frac{a}{d}(b c-a d)\right\}^{1 / 2} .
$$

They form the real two-dimensional vector space and we denote it by $N$.

Remark 1. The adjoint equation $D V=-{ }^{t} B V$ also has periodic solutions with the same periodicity as in Lemma 1, and they form the real two-dimensional vector space. We denote it by $N^{*}$.

Definition 1. We denote the projection which maps $D_{\sharp}^{\prime}$ onto $N$ (resp. $N^{*}$ ) by $P$ (resp. $Q$ ) and the null space of $P$ (resp. $Q$ ) by $P^{\perp}$ (resp. $\left.Q^{\perp}\right)$.

Lemma 2. The non-homogeneous linear equation

$$
D U=B U+H, \quad H \in D_{\#}^{\prime},
$$

has a solution in $D_{\#}^{\prime}$ if and only if $Q H=0$. Furthermore if $H$ satisfies this condition and belongs to $H_{\#}^{k}$, then there exists a unique solution of (5) in $H_{\#}^{k}$ which satisfies $P U=0$. If this unique solution is designated by $K H$, then $K$ is a linear operator mapping $H_{\#}^{k} \cap Q^{\perp}$ into $H_{\#}^{k} \cap P^{\perp}$, and there exists a positive constant $C$ such that $\|K H\|_{k} \leqq C\|H\|_{k}$.

To solve (3) we introduce the real parameters $\mu, \nu$ and new independent variables $x, y$ as follows :

$$
\xi=(1+\mu) x, \quad \eta=(1+\nu) y .
$$

Next we transform the dependent variable into $W={ }^{t}\left(w_{1}, w_{2}\right)$ as follows :

$$
U=W+U_{0}, \quad U_{0}={ }^{t}\left(u_{1}^{0}, u_{2}^{0}\right) \in N, \quad U_{0} \neq 0,
$$

where $N$ is the same space as in Lemma 1 in which $\xi, \eta$ are replaced by $x, y$, and $U_{0}$ is any periodic vector function from which periodic solutions of (3) will bifurcate. We denote the projections onto these $N$ and $N^{*}$ by the same notations, i.e., $P, Q$. Then $W$ satisfies the equation:

$$
D W=B W+G(x, y, W, \varepsilon, \mu, \nu),
$$

where

$$
\begin{aligned}
G= & \left(g_{1}, g_{2}\right), \\
g_{1}= & g_{1}\left(x, y, w_{1}, w_{2}, \varepsilon, \mu\right)=\mu\left(a\left(u_{1}^{0}+w_{1}\right)+b\left(u_{2}^{0}+w_{2}\right)\right) \\
& +(1+\mu) f_{1}\left(u_{1}^{0}+w_{1}, u_{2}^{0}+w_{2}, \varepsilon\right), \\
g_{2}= & g_{2}\left(x, y, w_{1}, w_{2}, \varepsilon, \nu\right)=\nu\left(c\left(u_{1}^{0}+w_{1}\right)+d\left(u_{2}^{0}+w_{2}\right)\right) \\
& +(1+\nu) f_{2}\left(u_{1}^{0}+w_{1}, u_{2}^{0}+w_{2}, \varepsilon\right) .
\end{aligned}
$$

Applying the projection $Q$ to (8), we obtain the system of equations 


$$
\begin{aligned}
D W & =B W+(I-Q) G \\
Q G & =0,
\end{aligned}
$$

where $I$ denotes the identity operator. This system is equivalent to (8). In the following two lemmas we state about the existence of solutions of (9.1) and their smooth dependency on the parameters. We use the following notation

$$
\begin{aligned}
C^{m}\left(R^{2} \times R^{1}, R^{2}\right)= & \left\{V={ }^{t}\left(v_{1}, v_{2}\right) ; v_{i}=v_{i}(x, y, \varepsilon)\right. \text { is a real-valued } \\
& m \text {-times continuously differentiable func- } \\
& \text { tion in } \left.R^{2} \times R^{1}, i=1,2\right\} .
\end{aligned}
$$

Lemma 3. Suppose that

$$
F(U, \varepsilon) \in C^{k+2}\left(R^{2} \times R^{1}, R^{2}\right), \quad k \geqq 2 ; \text { integer },
$$

and that positive constant $b$ is given. Then there exist positive constants $\varepsilon_{1}, \mu_{1}$ and $\nu_{1}$ such that for $\varepsilon, \mu, \nu,|\varepsilon| \leqq \varepsilon_{1},|\mu| \leqq \mu_{1},|\nu| \leqq \nu_{1}$ the equation

$$
W=K(I-Q) G(\cdot, \cdot, W, \varepsilon, \mu, \nu)
$$

has a unique solution $W(\varepsilon, \mu, \nu)$ which belongs to $H_{\#}^{k}(b) \cap P^{\perp}$ and is Lipschitz continuous with respect to $\varepsilon, \mu$ and $\nu$, and $W(0,0,0)=0$. This $W(\varepsilon, \mu, \nu)$ satisfies (9.1) in the sense of distribution.

Proof. Let us consider the following operator $T$

$$
T V=K(I-Q) G(\cdot, \cdot, V, \varepsilon, \mu, \nu) \text {. }
$$

Under the assumption of $F(U, \varepsilon)$ we can show that $T$ is a contraction mapping in $H_{\#}^{k}(b)$ for sufficiently small $\varepsilon, \mu, \nu$ with the aid of the lemmas of [3], [5], [6]. Then using the standard techniques, we can establish the lemma. More precisely see [4], [6], [7].

Remark 2. For $k \geqq 3, W(\varepsilon, \mu, \nu)$ is a classical solution of (9.1).

Lemma 4. Suppose that

$$
F(U, \varepsilon) \in C^{k+3}\left(R^{2} \times R^{1}, R^{2}\right), \quad k \geqq 2 ; \text { integer. }
$$

Then there exist positive constants $\varepsilon_{2}, \mu_{2}$ and $\nu_{2}\left(\varepsilon_{2} \leqq \varepsilon_{1}, \mu_{2} \leqq \mu_{1}, \nu_{2} \leqq \nu_{1}\right)$ such that for $\varepsilon, \mu, \nu,|\varepsilon|<\varepsilon_{2},|\mu|<\mu_{2},|\nu|<\nu_{2}, W(\varepsilon, \mu, \nu)$ in Lemma 3 has Fréchet derivatives from $R^{1}$ to $H_{\#}^{k}$ with respect to $\mu$ and $\nu$ which are continuous in operator norm.

Proof. See [4], [6], [7].

Next we solve the bifurcation equation (9.2). First we note (9.2) is equivalent to the system of equations

$$
\begin{aligned}
& B_{1}(\varepsilon, \mu, \nu)=\int_{0}^{T} \int_{0}^{S}{ }^{t} G \cdot \bar{V} d x d y=0, \\
& B_{2}(\varepsilon, \mu, \nu)=\int_{0}^{T} \int_{0}^{S}{ }^{t} G \cdot \bar{V}^{*} d x d y=0,
\end{aligned}
$$

where - denotes the usual inner product in $R^{2}, \bar{U}$ the complex conjugate vector of $U$, and $V$ and $V^{*}$ are appropriate bases in $N^{*}$. From Lemma 3 and the form of $G$, we easily see that $\varepsilon=\mu=\nu=0$ satisfies (12). Therefore we solve (12) with respect to $\mu$ and $\nu$ in the small neighbourhood of $\varepsilon=0$. The Jacobian of (12) at $\varepsilon=\mu=\nu=0$ is 
$\left.\frac{\partial\left(B_{1}, B_{2}\right)}{\partial(\mu, \nu)}\right|_{\epsilon=\mu=\nu=0}=\left|\begin{array}{ll}\int_{0}^{T} \int_{0}^{S}\left(a u_{1}^{0}+b u_{2}^{0}\right) \bar{v}_{1} d x d y, & \int_{0}^{T} \int_{0}^{S}\left(c u_{1}^{0}+d u_{2}^{0}\right) \bar{v}_{2} d x d y \\ \int_{0}^{T} \int_{0}^{S}\left(a u_{1}^{0}+b u_{2}^{0}\right) \bar{v}_{1}^{*} d x d y, & \int_{0}^{T} \int_{0}^{S}\left(c u_{1}^{0}+d u_{2}^{0}\right) \bar{v}_{2}^{*} d x d y\end{array}\right|$,

where $V={ }^{t}\left(v_{1}, v_{2}\right)$ and $V^{*}={ }^{t}\left(v_{1}^{*}, v_{2}^{*}\right)$.

Using the hypotheses of (2) and $U_{0} \neq 0$, we can easily see that (13) is not equal to zero. By the implicit function theorem we obtain the following lemma.

Lemma 5. Under the hypotheses of (2), $U_{0} \neq 0$ and (11), there exist positive constants $\varepsilon_{3}, \mu_{3}, \nu_{3}\left(\varepsilon_{3} \leqq \varepsilon_{2}, \mu_{3} \leqq \mu_{2}, \nu_{3} \leqq \nu_{3}\right)$ such that (12) has a unique one-parameter family of solutions $\mu(\varepsilon), \nu(\varepsilon)$ for $|\varepsilon| \leqq \varepsilon_{3},|\mu| \leqq \mu_{3}$, $|\nu| \leqq \nu_{3}$, and which are continuous in $\varepsilon$ and $\mu(0)=\nu(0)=0$.

Using Lemmas 1-5 and transformations (6), (7), we conclude:

Theorem. Suppose that $F(U, \varepsilon)$ satisfies $F(U, 0)=0$ and $(11), B$ satisfies (2), and that $a_{1}, b_{1}\left(0<a_{1}<b_{1}\right)$ are given constants. Then for any $U_{0}(\neq 0) \in N,\left\|U_{0}\right\|_{k} \leqq a_{1}$, there exists a positive constant $\varepsilon_{0}=\varepsilon_{0}\left(a_{1}, b_{1}\right.$, k) such that for $|\varepsilon| \leqq \varepsilon_{0}$ the equation (3) has a unique one-parameter family of periodic solutions $U(\varepsilon)$ with the properties;

1) $\|\tilde{U}(\varepsilon)\|_{k} \leqq b_{1}$, where $\tilde{U}(\varepsilon)=\tilde{U}(x, y, \varepsilon)$

$$
=U((1+\mu(\varepsilon)) x,(1+\nu(\varepsilon)) y, \varepsilon),
$$

2) $(1+\mu(\varepsilon)) S$-periodic in $\xi$, and $(1+\nu(\varepsilon)) T$-periodic in $\eta$,

3) $\tilde{U}(\varepsilon)$ tends to $U_{0}$ as $\varepsilon \rightarrow 0$ in $H_{\#}^{k}$-norm sense, where $\mu(\varepsilon)$ and $\nu(\varepsilon)$ are uniquely defined continuous functions for $|\varepsilon| \leqq \varepsilon_{0}$ and $\mu(0)=\nu(0)=0$.

Example. Let us consider the system of equations

$$
\begin{aligned}
& \partial u_{1} / \partial t+c_{1}\left(\partial u_{1} / \partial x\right)=\left(\gamma_{1}-a u_{1}-b u_{2}\right) u_{1}, \\
& \partial u_{2} / \partial t+c_{2}\left(\partial u_{2} / \partial x\right)=\left(\gamma_{2}-c u_{1}-d u_{2}\right) u_{2},
\end{aligned}
$$

where all coefficients of the right-hand side are positive and satisfy the following conditions

$$
a d-b c<0, \quad\left(\gamma_{2} / c\right)<\left(\gamma_{1} / a\right), \quad\left(\gamma_{1} / b\right)<\left(\gamma_{2} / d\right) .
$$

This system describes the behavior of the populations of two species $u_{1}$ and $u_{2}$ which compete with each other and migrate in different directions (cf. [8]).

From the conditions (15), we easily see that (14) has a positive constant equilibrium solution

$$
\left(\hat{u}_{1}, \hat{u}_{2}\right)=\frac{1}{a d-b c}\left(d \gamma_{1}-b \gamma_{2}, a \gamma_{2}-c \gamma_{1}\right) .
$$

If we apply the following transformation to (14)

$$
\varepsilon v_{1}=u_{1}-\hat{u}_{1}, \quad \varepsilon v_{2}=u_{2}-\hat{u}_{2}, \quad \varepsilon \text {; real parameter, }
$$

we obtain the perturbed equations from the equilibrium solution (16) as follows :

$$
\partial v_{1} / \partial t+c_{1}\left(\partial v_{1} / \partial x\right)=-a \hat{u}_{1} v_{1}-b \hat{u}_{1} v_{2}-\varepsilon\left(a v_{1}^{2}+b v_{1} v_{2}\right)
$$




$$
\partial v_{2} / \partial t+c_{2}\left(\partial v_{2} / \partial x\right)=-c \hat{u}_{2} v_{1}-d \hat{u}_{2} v_{2}-\varepsilon\left(d v_{2}^{2}+c v_{1} v_{2}\right)
$$

From the first condition of (15) we can apply Theorem to this system of equations.

\section{References}

[1] O. Vejvoda: Periodic solutions of a linear and weakly nonlinear wave equation in one dimension. I. Czech. Math. J., 14, 341-382 (1964).

[2] L. Cesari: Existence in the large of periodic solutions of hyperbolic partial differential equations. Arch. Rational Mech. Anal., 20, 170-190 (1965).

[3] J. Moser: A rapidly convergent iteration method and nonlinear partial differential equations. Ann. Scuola Norm. Super. Pisa, Ser. 3, 20, 265-315. (1966).

] 4 [ J. K. Hale: Periodic solutions of a class of hyperbolic equations containing a small parameter. Arch. Rational Mech. Anal., 23, 380-398 (1967).

[5] P. H. Rabinowitz: Periodic solutions of nonlinear hyperbolic partial differential equations. Comm. Pure Appl. Math., 20, 145-205 (1967).

[6] W. S. Hall: Periodic solutions of a class of weakly nonlinear evolution equations. Arch. Rational Mech. Anal., 39, 294-322 (1970).

[7] Y. Nishiura: Master thesis, Faculty of Science, Osaka Univ. (1975) (in Japanese).

[8] V. Volterra: Leçon sur la théorie mathématique de la lutte pour la vie. Gauthier-Villars, Paris (1931). 